\title{
LES MÉTHODES TRADITIONNELLES DE CONSERVATION DES ALIMENTS ET L'ENJEU HISTORIQUE ET GÉOPOLITIQUE DE LA SUFFISANCE ALIMENTAIRE
}

\author{
TRADITIONAL METHODS OF FOOD PRESERVATION AND THE \\ HISTORICAL AND GEOPOLITICAL CHALLENGE OF FOOD SUFFICIENCY
}

Par Richard BONNE ${ }^{1}$

(Manuscrit reçu le 22 mars 2021 et accepté le 15 juillet 2021)

\begin{abstract}
RÉSUMÉ
Depuis toujours, assurer sa suffisance alimentaire a été un challenge crucial pour l'humanité. Bien que les ressources alimentaires soient abondantes, la difficulté fréquente pour y accéder, qui venait de leur éloignement ou de leur saisonnalité, n'a pu être surmontée que par des méthodes de conservation conférant aux aliments une composition résistant à l'altération microbienne. Des rivalités géopolitiques sont nées de la compétition pour accéder à ces ressources. Cet article expose le mécanisme de fonctionnement des méthodes traditionnelles de conservation et décrit les produits obtenus ainsi que le principal risque sanitaire généré par une alimentation dépourvue de produits frais : le scorbut. Des données historiques sont rassemblées sur quelques produits ainsi que sur les conséquences géopolitiques nées des rivalités pour accéder à ces ressources alimentaires. Sur le marché mondialisé contemporain, les méthodes et les objectifs de la conservation des aliments ont bien changé, cependant le contexte conflictuel international perdure.
\end{abstract}

Mots-clés : suffisance alimentaire, méthodes de conservation, données historiques, rivalités géopolitiques, scorbut

\begin{abstract}
Ensuring food security has always been a crucial challenge for humanity. Although food resources are abundant, the frequent difficulty in accessing them, which arose from their remoteness or seasonality, could only be overcome by preservation methods that give food a composition resistant to microbial deterioration. Geopolitical rivalries have arisen from the competition for access to these resources. This article explains the mechanism of operation of traditional methods of conservation and describes the products obtained as well as the main health risk generated by a diet devoid of fresh food: the scurvy. Historical data is gathered on some products as well as on the geopolitical consequences arising from rivalries for access to food resources. In the contemporary globalized market the methods and objectives of food preservation have changed a lot, however the international context of conflict persists.

Key-Words: Food security, preservation methods, historical data, geopolitical rivalries, scurvy.
\end{abstract}

1- DMV, PhD, Expert International en Sécurité Sanitaire des Aliments - 13, rue Caumont, 32000 Auch, France. Courriel : bonne.richard2@orange.fr 


\section{LISTE DES ACRONYMES}

ASEAN: The Association of Southeast Asian Nations CIPV : Convention Internationale pour la Protection des Végétaux FAO: Food \& Agriculture Organization

HACCP: Hazard Analysis Critical Control Point

HMS: Her/His Majesty Ship

OIE: Office International des Épizooties

OMC : Organisation Mondiale du Commerce

OMS : Organisation Mondiale de la Santé

PMS : Plan de Maîtrise Sanitaire

UE : Union Européenne

\section{INTRODUCTION}

Depuis l'aube des temps, garantir au quotidien sa suffisance alimentaire et donc sa survie, en écartant le spectre de la famine, a été un enjeu crucial pour l'humanité. Une analyse plus fine de cette problématique montre que des périodes de déficit alimentaire, responsables des famines, apparaissaient périodiquement bien que la ressource alimentaire soit souvent globalement abondante. Cependant, cette réserve n'était pas directement ou constamment accessible.

\section{Situations où le recours à la conservation des aliments s'impose}

Soit la ressource était abondante mais lointaine. Pour illustrer cette situation nous développerons l'exemple de la morue pêchée sous les hautes latitudes de l'Atlantique Nord, participant dès le XVI ${ }^{\text {ème }}$ siècle à la couverture des besoins alimentaires de l'Europe occidentale.

Soit la ressource était abondante mais saisonnière. C'est le cas de la production des femelles laitières domestiques, liée au cycle des saisons, transformée en fromage, ou bien des salmonidés migrateurs de rivière, salés et fumés, ou bien encore de la cueillette estivale des baies sauvages et des fruits, accommodés en pâtes ou confitures.

Soit la ressource devait accompagner les déplacements au loin. Cette problématique est propre au mode de vie des populations nomades ou aux voyages au long cours, mais aussi à l'organisation d'expéditions guerrières. Pour illustrer ces trois situations, peuvent être donnés comme exemples : le biscuit de marine des voyages transocéaniques du XV'me au XVIII ${ }^{\text {ème }}$ siècle, le pemmican des populations amérindiennes puis enfin celui des conserves appertisées des campagnes napoléoniennes.

Soit la préservation de la ressource alimentaire devait être indépendante $d u$ cycle des saisons. Peuvent être citées pour cette dernière éventualité : la conservation des viandes par la salaison sèche ou en saumure, la choucroute et enfin l'enfouissement des viandes dans la neige.

\section{Avantages des méthodes traditionnelles de conservation des aliments}

Il ressort de façon évidente des lignes qui précédent, que la mise en œuvre de méthodes de conservation des aliments, découvertes en observant les phénomènes naturels, ou bien inventées, devenait une condition incontournable pour garantir au quotidien la suffisance alimentaire. À l'usage, ces méthodes de conservation ont montré qu'elles étaient génératrices de plusieurs avantages.

Préservation de la salubrité des aliments. Elles préservent la salubrité des aliments dont la consommation peut être différée sans causer de troubles au consommateur.

Préservation de la valeur économique des aliments. Elles confèrent aux aliments conservés des caractéristiques nutritives et gustatives agréables, indispensables à leur consommation (goût, odeur, texture, ...), et évitent ainsi les pertes de valeur économique. De plus, la haute valeur économique de certains des aliments conservés, permet d'en faire des biens d'échange marchand avec d'autres populations.

Obtention de nouveaux aliments. L'obtention d'aliments significativement différents de la matière première mise en œuvre (flaveur, texture), comme les fromages et le yaourt obtenus à partir du lait, ou les salaisons préparées à partir de viandes, permet d'enrichir le régime alimentaire avec de nouveaux éléments, présentant pour certains, comme la choucroute, un réel intérêt nutritionnel. Cependant il est établi par la médecine, que le recours prolongé à un régime alimentaire totalement dépourvu de produits frais, génère des troubles pathologiques graves, provoqués par des carences vitaminiques. Il n'est donc pas possible de développer cette présentation sur quelques méthodes traditionnelles de conservation des aliments sans avoir abordé préalablement l'aspect carentiel, au premier rang duquel il faut citer le scorbut, induit par la pauvreté en vitamine $\mathrm{C}$ d'un régime alimentaire composé exclusivement de ces produits.

Les généralités qui viennent d'être exposées, pourraient à elles seules justifier l'intérêt porté au problème de la suffisance alimentaire qui dépend de l'accès aux ressources alimentaires et des méthodes permettant d'en étendre la consommation dans le temps et/ou dans l'espace. Mais le champ de cette étude peut encore être élargi. En effet, les rivalités stratégiques et économiques pour accéder à ces ressources ont aussi façonné de façon durable les relations géopolitiques des États Européens et de leurs empires coloniaux. Le XVII ${ }^{\text {ème }}$ siècle s'est par exemple illustré par un véritable conflit autour de la morue opposant l'Angleterre à la France et à d'autres pays maritimes de la façade atlantique. Comme nous le verrons plus loin, avec ce premier conflit maritime, l'Angleterre a chassé les pêcheurs des pays concurrents de la zone nord-atlantique et a compris l'intérêt d'instaurer sa domination navale sur l'ensemble des mers et 
océans du globe. Cette domination maritime a ensuite perduré pendant plus de trois siècles. Compte tenu de la vaste étendue de son volet géopolitique, cet article, après avoir mentionné les principales méthodes traditionnelles de conservation des aliments et le risque de scorbut induit par leur emploi, se limitera au développement de deux exemples d'intérêt historique : la morue salée et le pemmican des amérindiens.

\section{ÉLÉMENTS DE BASE SUR L'ALTÉRATION DES ALIMENTS}

\section{Besoins nutritifs communs aux microorganismes et à l'espèce humaine}

Les microorganismes, bactéries et champignons (levures et moisissures), viennent de très loin au premier rang des agents responsables de la dégradation des aliments, tant des altérations organoleptiques qui leur font perdre tout ou partie de leur valeur économique en les rendant inconsommables, que des toxi-infections alimentaires. Les altérations biochimiques, telles que le rancissement, n'ont pas été prises en compte par les méthodes de conservation traditionnelles et ont même été souvent considérées comme normales, preuve en est, par exemple, la large consommation de beurre rance en Asie centrale. Depuis la fin du XIX ${ }^{\text {ème }}$ siècle, l'essor de la microbiologie a permis d'identifier les besoins nutritifs des microorganismes, ce qui s'est traduit en pratique par la production de milieux de culture. L'examen de la composition d'un milieu de culture usuel, comme par exemple une gélose pour numération, montre qu'elle se rapproche de celle des aliments frais et aussi de nombreuses préparations culinaires (plats cuisinés, sauces, pâtisseries, salades composées ...). Une erreur de dosage d'un composant, en plus ou en moins, dans la préparation d'un milieu de culture peut le rendre impropre au développement des microorganismes. C'est le cas par exemple d'une concentration trop faible en eau ou trop forte en sel ou en saccharose ou encore en lipides. Cet effet dû à un déséquilibre par rapport à la composition des milieux de culture usuels, a été utilisé depuis la préhistoire, de façon empirique, pour stabiliser et conserver les aliments, c'est-à-dire pour maintenir leurs qualités organoleptiques, ou leur en conférer de nouvelles qui soient acceptables, tout en préservant leur innocuité. Cette stratégie portant sur la composition des aliments, a donné, par exemple, naissance aux aliments secs pauvres en eau, aux salaisons riches en sels, aux confitures à forte teneur en saccharose et aussi aux confits de viande saturés en lipides.

Les besoins nutritifs des microorganismes et de l'espèce humaine sont donc très comparables. Si ces aliments, conservés par les méthodes traditionnelles portant sur leur composition, sont impropres au développement des microorganismes, ils ne peuvent pas non plus, sur le long terme, couvrir tous les besoins nutritifs des consommateurs. En effet, compte tenu de leur pauvreté en vitamines, leur consommation exclusive induit un régime alimentaire déséquilibré, responsable de carences sévères provoquant des troubles graves souvent mortels. L'essor de la microbiologie a aussi montré l'influence de la température sur la vitesse de développement des microorganismes, mais l'effet de ralentissement des altérations par le froid, n'a été que très peu appliqué, faute d'avoir été clairement perçu avant l'époque moderne. De plus, sortis de leurs " trous de neige ", les aliments congelés s'altéraient très rapidement et en conséquence ne pouvaient être transportés sur de longues distances.

\section{Définition de trois paramètres biochimiques à prendre en compte}

L'aw (ou activité hydrique.) Elle constitue l'expression affinée et chiffrée de la disponibilité en eau libre d'un milieu nutritif, nécessaire à la multiplication bactérienne. Cette valeur est différente du pourcentage global en eau, car elle tient compte de la fixation de l'eau par certaines molécules : sucre, sel.

Le pH (ou potentiel hydrogène). C'est l'expression chiffrée de "l'acidité/neutralité/alcalinité " d'un milieu nutritif. Il est possible, pour chaque germe, de définir des plages de valeurs de $\mathrm{pH}$ dans lesquelles sa multiplication est possible ou pas. Les basses valeurs de $\mathrm{pH}$, retenues pour la conservation de certains aliments, inhibent le développement des germes pathogènes et d'altération, tandis que l'activité favorable des flores lactiques se poursuit.

La teneur en lipides. Le beurre, les huiles végétales et les graisses animales sont connus pour être peu sensibles à la dégradation par les microorganismes (levures, moisissures, bactéries) et pour ne s'altérer principalement que par voie biochimique souvent initiée par des enzymes : le rancissement.

\section{Les accidents alimentaires}

Ces accidents alimentaires peuvent être de deux ordres : sanitaires, se traduisant par des toxi-infections alimentaires, ou économiques, générateurs de pertes. Leur commun mécanisme d'apparition fait intervenir trois composantes, que sont la contamination, la multiplication et la survie (Bonne, 2013a). Jusqu'à l'essor de la microbiologie, le monde des microorganismes était totalement inconnu et en conséquence, aucune mesure de propreté visant à réduire la contamination, n'était mise en ouvre. Pour la même raison, les traitements de décontamination des aliments, visant à réduire ou éliminer leur flore microbienne et donc s'opposer à sa survie, étaient complétement inconnus. C'est avec la découverte de l'appertisation (Frèrejean, 2010) à la toute fin du XVIII ${ }^{\text {ème }}$ siècle (1795), qu'une méthode de décontamination a montré son efficacité, sans pour autant que la raison en soit connue. De même avant cette date, l'effet inhibiteur du froid étant mal connu, la quasi-totalité des méthodes de conservation appliquées, à l'exception de celle des viandes dans des "trous de neige», consistaient à produire des aliments dont la composition biochimique soit défavorable au développement des microorganismes. 


\section{LE SCORBUT OU CARENCE SÉVÈRE EN VITAMINE C, VÉRITABLE FLÉAU DES VOYAGES TRANSOCÉANIQUES AU LONG COURS}

Avant le XIX ${ }^{\text {ème }}$ siècle, le scorbut est le principal facteur de risque s'opposant à la consommation sur une longue période d'une alimentation dépourvue de produits frais et donc des voyages transocéaniques au long cours. Cette carence a eu une telle importance qu'il est nécessaire d'en décrire les caractéristiques et les conséquences géopolitiques avant de poursuivre cet exposé.

\section{Étiologie et symptomatologie}

Le scorbut est provoqué par la carence sévère en vitamine C. Il est à l'origine de symptômes (Berche, 2019) dont la chronologie d'apparition se décline comme suit : douleurs articulaires et asthénie, puis gingivite inflammatoire et hémorragique suivie de la chute des dents, et enfin syndrome hémorragique cutané, œedème des membres inférieurs et dyspnée, précédant la mort dans une extrême faiblesse (Sweg, 1938).

\section{Quelques éléments historiques sur le scorbut}

Le scorbut est peu connu avant le XV ${ }^{\text {ème }}$ siècle, sauf dans les villes assiégées (Le Songeux, 1827) où la population est privée durablement de nourriture fraîche. Avant cette époque on ne pratique que la navigation de cabotage, avec de fréquentes escales à terre, assurant un apport régulier de nourriture fraîche et donc en vitamine C. L'impact du scorbut devient de plus en plus important du XV'ème au XVIII ${ }^{\text {ème }}$ siècle avec l'allongement des traversées maritimes. L'alimentation des marins se compose sur de longues périodes, de biscuits et de salaisons. Les grands voiliers hollandais quittant l'Europe pour se rendre en Extrême Orient (Malaisie, Indonésie ...), se déplacent à une vitesse moyenne ne dépassant pas 4 à 5 kilomètres par heure, ce qui impose de naviguer sans escale pendant plusieurs mois (Leys, 2005). L'explorateur français Jacques Cartier (1491-1557), lors de son voyage de 1536 au cours duquel il remonte l'estuaire du Saint-Laurent et y hiverne, perd en chemin 25 marins sur un équipage de 110 , en raison du scorbut, tandis que 40 en sont gravement malades à leur arrivée. Heureusement, les matelots de Jacques Cartier vont tous se rétablir, car les amérindiens de la nation algonquienne qui peuple la région, savent reconnaître et traiter le scorbut par l'administration de tisane de feuilles et d'écorce d'anedda (Thuya occidentalis) (Fonssagrives, 1877). L'expédition maritime de Magellan (1519-1522), dont un des trois navires est le premier à boucler un tour du monde, perd pour sa part 245 marins, principalement du scorbut, sur un équipage de 265 au départ (Sweg, 1938).

\section{Mise en place progressive de mesures de prophylaxie}

Au début du XVII ìme siècle, on comprend que la situation s'améliore aux escales par la consommation d'aliments frais (légumes et agrumes). Vers 1660, la Compagnie Néerlandaise des Indes Orientales établit un point d'avitaillement au Cap, qui est le premier point du voyage sur la route des Indes Hollandaises où l'on trouve des conditions climatiques comparables à celles de l'Europe. Les hollandais y élèvent du bétail et y cultivent des légumes et des fruits européens, pour avitailler en produits frais les équipages de leurs bateaux qui y font relâche. Ils y implantent aussi la culture de la limette, sorte de citron vert et fruit d'un arbre du genre Citrus (C. limetta), dont les effets préventifs et curatifs sur le scorbut ont été pressentis. La culture de la limette est également introduite à cette époque sur les iles Maurice et Ste Hélène. En 1747, un médecin de marine, James Lind (1716-1794) met en évidence pour la "Royal Navy ", la prévention du scorbut grâce aux fruits frais (agrumes) ou au cidre, pourvoyeurs de vitamine $\mathrm{C}$ dans la ration des matelots. $\mathrm{Au}$ cours d'une traversée transocéanique, il réalise un véritable essai clinique en administrant des fruits frais à une partie de l'équipage, du cidre à une seconde, la dernière ne recevant rien pour faire office de lot témoin. A l'arrivée, les observations faites sur les trois groupes de marins, démontrent sans appel, l'effet de prévention du scorbut par les fruits frais et le cidre. James Lind affine son expérimentation en montrant l'efficacité du jus de citron, qu'il échoue cependant à stabiliser par la chaleur (Portail pédagogique de l'Académie de Toulouse, 2021). A partir de 1795, l'adjonction de 10\% d'alcool au jus de citron règle le problème et cette formule reste "Secret Défense» jusqu'en 1840, la "Royal Navy " considérant que cette méthode protégeant ses équipages du scorbut, participe au maintien de sa domination sur les océans du monde. Après ces découvertes, la production massive de citrons est lancée en Sicile, dont le climat est favorable à cette culture. Ce fruit, que beaucoup de pays réclament (Europe, USA) pour leur marine à voile, devient un véritable or jaune pour la Sicile. Cette ile aux structures politiques encore féodales, est dominée par des grands propriétaires terriens, employant une paysannerie extrêmement pauvre. Les dispositions du maintien de l'ordre dans le Royaume des Deux-Siciles, sont quasiment inexistantes à cette époque et le vol des récoltes de citrons prend rapidement des proportions mettant en péril cette activité économique naissante. En guise de réponse, les propriétaires engagent alors des escouades d'hommes de main pour juguler le vol. Malheureusement avec le temps, ces vigiles, engagés pour protéger les domaines, s'organisent et prennent de plus en plus de pouvoir dans l'île jusqu'à constituer la mafia (Olsen, 2018). Cette évolution des rapports de force dans l'île montre, qu'au plan géopolitique, l'émergence d'une source de richesse économique d'importance majeure, dans un pays pauvre doté d'un pouvoir politique faible, conduit immanquablement à la corruption et à la violence. Enfin, après la découverte de l'appertisation en 1795 (Frèrejean, 2010), la France opte en 1804 pour cette technique de conservation des aliments, afin d'assurer l'avitaillement des équipages de ses navires. Elle participe à une alimentation équilibrée, où les vitamines sont conservées, faisant reculer le scorbut.

\section{PRINCIPAUX MÉCANISMES DE CONSERVA- TION ADOPTÉS PAR LES MÉTHODES TRADI- TIONNELLES}

\section{Conservation par la réduction de l'activité d'eau $\left(\mathrm{a}_{\mathrm{w}}\right)$}

Généralités sur l'activité d'eau ou "Activity of Water ». La formule de calcul de l'aw d'un aliment est la suivante $\mathrm{aw}=\mathrm{Pw} / \mathrm{Pw}^{\circ}$ dans laquelle $\mathrm{Pw}$ est la pression partielle de vapeur saturante du produit et $\mathrm{Pw}^{\circ}$ la pression de vapeur saturante de 
l'eau pure à la même température (Oteng-Gyang, 1984). La valeur de l'aw est comprise entre 0 et 1 . La valeur de l'aw est égale à 1 pour l'eau pure $\left(\mathrm{aw}=\mathrm{Pw}^{\circ} / \mathrm{Pw}^{\circ}\right)$, tandis qu'une aw $<0,50$ ne permet plus d'observer une quelconque activité métabolique microbienne (Tableau I).

\begin{tabular}{|ll}
\hline Valeurs d' aw & $\begin{array}{l}\text { Les micro-organismes sont inhibés par la } \\
\text { limite inférieure d' aw }\end{array}$ \\
\hline aw $=1,00-0,95$ & $\begin{array}{l}\text { Bactéries Gram-négatives } \\
\text { Nombreuses bactéries Gram-positives, } \\
\text { Clostridium botulinum }\end{array}$ \\
\hline aw $=0,95-0,91$ & Nombreuses levures \\
\hline aw $=0,87-0,87$ & $\begin{array}{l}\text { Nombreuses moisissures, Staphylococcus } \\
\text { aureus }\end{array}$ \\
\hline aw $=0,80-0,75$ & Bactéries halophiles \\
\hline aw $=0,75-0,65$ & Moisissures xérophiles \\
aw $=0,65-0,60$ & Levures osmophiles
\end{tabular}

Tableau I : Relations entre l'activité de l'eau (aw) et les microorganismes (Oteng-Gyang, 1984).

Une grande part des méthodes de conservation traditionnelles des aliments, est basée sur l'abaissement de cette valeur, soit en déshydratant les produits par séchage, soit par l'adjonction de sel ou de sucre ayant le pouvoir de fixer les molécules d'eau, comme dans les confitures ou les produits en saumure, soit enfin en combinant ces deux mécanismes pour la morue ou les salaisons sèches. Pour certains produits comme les viandes en saumure, une opération complémentaire de fumage peut renforcer l'effet de conservation.

La morue : un exemple de produit conservé par salaison. La salaison, pratiquée au sel sec ou en saumure, est un exemple où l'effet recherché est une conservation du produit qui soit indépendante des variations climatiques. Le vaste domaine des salaisons pourrait faire à lui seul l'objet d'une publication spécifique.

Nature $d u$ produit. La morue est un exemple, dans lequel la ressource alimentaire est très périssable et abondante mais lointaine. La morue est un poisson qui vit en profondeur mais qui remonte au printemps ou en été, selon les régions, pour frayer sur des bancs en eau peu profonde. C'est pendant cette période de reproduction qu'a lieu la campagne de pêche. Nous avons choisi de développer largement l'exemple de la morue salée, car les conséquences des conflits entrainés au XVII ${ }^{\text {ème }}$ siècle pour l'accès à cette ressource halieutique, ont encore des répercussions géopolitiques à notre époque. Le produit connu sous l'appellation de "morue salée " est majoritairement le produit transformé par salage et séchage de la morue ou cabillaud (Gadus morhua). Cependant, d'autres espèces de poissons peuvent être utilisées pour sa production : la morue du Groenland (Gadus ogac), la morue boréale (Eliginus gracilis)... Le terme de «cabillaud " est généralement attribué au poisson frais, tandis que celui de " morue » est plutôt réservé au produit salé et séché, dont la conservation est assurée par une valeur d'aw inférieure à 0,91 .
Quelques données historiques. Aux XI ${ }^{\text {ème }}$ et XII ${ }^{\text {mè }}$ siècles, cette pêche est exclusivement norvégienne (Bernet, 1998). La pêche a alors lieu sur les côtes d'Europe occidentale (dans le secteur maritime de Doggers Bank) dont la ressource limitée finit par ne plus satisfaire la demande. En 1443, Terre-Neuve et la Nouvelle Écosse apparaissent sur un portulan portugais (carte maritime en usage à cette époque) et vers 1450 , la pêche à la morue fait déjà l'objet d'une activité intense. Mais il est très probable que l'activité de pêche dans cette zone est plus ancienne et remonte au XIV ème et peut-être même au XIII ${ }^{\text {ème }}$ siècle (Soyez, 1981). On sait avec certitude que, vers l'an 1000, le chef viking Eric le Rouge, probablement proscrit de Scandinavie, a fondé une colonie au Groenland. Les vikings utilisent, à cette époque, la morue salée comme aliment de voyage bien que la production de sel soit difficile en Scandinavie. Eric le Rouge (ou son fils), pousse ensuite sa navigation plus loin vers l'ouest jusqu'aux côtes nord-américaines, première région où pousse la vigne sauvage, à laquelle il donne le nom de "Vinland ». Depuis, ce premier indice historique a été complété par la découverte de restes de constructions côtières vikings et de vestiges appartenant à la culture nordique sur des sites archéologiques amérindiens, attestant d'échanges avec les populations autochtones. Un autre indice conforte l'hypothèse selon laquelle, au début du XVI ème siècle, les amérindiens étaient déjà coutumiers de contacts avec les européens. Lorsqu'en 1534 Jacques Cartier remonte l'estuaire du Saint-Laurent pour la première fois, des indiens Micmacs se portent à sa rencontre en gesticulant à bord de pirogues. Outre le fait qu'elle soit à l'origine de l'expression "faire des micmacs ", cette rencontre est une nouveauté pour Cartier mais n'en est pas une pour les indiens. La preuve en est que leurs embarcations sont chargées de marchandises de traite, en l'occurrence des pelleteries, qu'ils ont, à n'en pas douter, déjà pris l'habitude d'échanger avec les équipages des navires de pêche européens (Soyez, 1981). Les données historiques qui précèdent, montrent que, dès cette époque, l'existence de zones de pêche à la morue en Amérique du Nord, est connue des marins d'Europe occidentale. Cependant, cette réalité s'oppose à la conception de l'Église catholique pour laquelle la terre est plate, ce qui exclut la présence de terres à l'ouest. Pour un temps, l'Église catholique feint d'ignorer l'activité de pêche dans cette zone, mais au XV ${ }^{\text {ème }}$ siècle ce secret de Polichinelle est de moins en moins bien gardé. Cette politique tient au fait que, jusqu'à la fin du XVème siècle, la priorité de la papauté est d'achever la "Reconquista " de la péninsule ibérique et qu'elle impose, pour ce faire, aux royaumes catholiques, de concentrer tous leurs moyens sur cet objectif au détriment de tout autre. Le royaume maure de Grenade tombe le 2 janvier 1492, ce qui libère les royaumes catholiques de leur engagement de reconquête et permet à Christophe Colomb, le 12 octobre 1492, de poser le pied sur une ile des Bahamas dont il prend possession au nom des rois catholiques, Isabelle de Castille et Ferdinand d'Aragon, qui ont financé l'expédition. En 1508 la morue représente $10 \%$ du poisson commercialisé au Portugal. Une activité économique, d'une telle importance pour le Portugal, n'a pas pu se développer dans la décennie qui a suivi le premier voyage de Christophe Colomb, elle était donc très 
probablement antérieure à cet évènement. Au début du XVİ̀me siècle, les Basques, les Français et les Portugais pêchent en relativement bonne intelligence (Delâge, 1985) sur «le banc de Terre-Neuve» tandis que les Anglais n'ont pas d'activité dans cette zone et ne viendront que plus tard, dans les années 1620 , mais à bord de navires armés. En effet, l'Angleterre protestante n'a pas encore une flotte importante et surtout, elle n'a pas, pour sa consommation, les besoins conséquents en poisson des pays catholiques, qui doivent respecter au cours de l'année de nombreux « jours maigres " imposés par leur Église. La morue salée permet à ces pays, d'étendre la consommation régulière et sans risque de poisson à l'intérieur des terres, loin des côtes où il est pêché. L'économie de la morue concerne aussi les régions méditerranéennes, où le produit fini est échangé contre du sel des salines locales nécessaire à la production. La tradition de la "brandade de morue de Nîmes " demeure un témoin de ces échanges. à partir de 1620 , consciente du retard qu'elle a pris dans l'exploitation de cette ressource économique majeure, l'Angleterre impose alors rapidement sa domination sur les zones nord-atlantiques de pêche à la morue (Barré, 2005). Ce premier succès maritime, obtenu par la force, est le point de départ de sa politique de domination maritime des mers et océans du globe. Enfin, avec la convention anglo-française de 1904, la France perd presque tous ses droits de pêche à la morue dans cette partie du Monde.

Le mode de pêche. Deux principaux modes de pêches sont pratiqués au XVII ${ }^{\text {ème }}$ siècle (Hersart de la Villemarqué, 1995) et leur choix va jouer un rôle crucial dans l'éviction de certains pays de la zone de pêche. La "pêche sédentaire ", d'une part, permet la production de la "morue séchée ou blanche " de haute qualité, très stable. Des chaloupes partent quotidiennement à la pêche et au retour, à terre, le poisson est fileté, salé au sel sec. Puis, après brossage, il est mis à sécher sur des " échafauds " de bois ou à terre, sur des aires empierrées appelées " graves ». D'autre part, la « pêche errante " permet la production de «morue verte " de moindre qualité. Les navires pêchent en dérivant sur les bancs de Terre-Neuve et en suivant les mouvements des bancs de poissons. Ils mettent aussi des doris à la mer sur lesquels on embarque deux hommes, un chien de race "Terre-Neuve " ainsi qu'une réserve d'eau et de biscuits de marine. Le poisson est fileté, salé et partiellement séché sur le pont du navire avant d'être mis en soute. Au XVII ${ }^{\text {ème }}$ siècle, les pêcheurs français pratiquent majoritairement la pêche errante ce qui les rend très vulnérables aux attaques des nombreux bateaux anglais. Les Français ont établi des colonies sur les rives du Saint-Laurent, qui pourraient leur servir de position de repli, mais la marine anglaise bloque l'entrée de l'estuaire du fleuve. Par ailleurs, la flotte française ne peut pas leur venir en aide car elle est de piètre qualité et surtout elle est retenue, à cette époque et jusqu'en 1629, par le siège de La Rochelle. Au XVII ${ }^{\text {ème }}$ siècle, la bataille de la morue est très mal engagée pour la France face à l'Angleterre. Elle est définitivement perdue en 1763 avec le traité de Paris et la fin du Canada français.

Le biscuit de marine. En se référant aux différentes situations énoncées en introduction, le biscuit de marine est un exemple, dans lequel la ressource alimentaire doit être emportée pour permettre de voyager au loin. C'est un pain à la farine de froment, sans levain, sans sel, très dense, d'un diamètre de 20 $\mathrm{cm}$ (Clouet, 2009). Il subit une cuisson double (1h30), d'où le nom de "biscuit ", puis un ressuage de plusieurs semaines, ce qui lui confère une valeur finale d'aw très basse de l'ordre de 0,60 . Très dur, sa consommation est rendue difficile par la dentition en mauvais état des marins atteints de scorbut. Il est conservé en soute étanche, car, en mer, il est très vulnérable à la réhydratation qui favorise sa dégradation par les moisissures et les insectes. Le biscuit de marine est déjà utilisé sous l'antiquité (Panis nauticus). Il est réintroduit en France pour la Marine Royale par François I ${ }^{\text {er }}(1494$ - 1547) en 1543 et devient, avec les salaisons en saumure, la base de l'avitaillement de la marine à voile.

Les confitures. Dans le cas des confitures, les fruits constituent une ressource saisonnière, abondante mais très altérable. Elles sont un mélange, à parts égales, de fruits et de sucre, cuit et déshydraté dans un récipient préférablement en cuivre, dont la valeur d'aw est comprise entre 0,91 et 0,60 en fonction de leur teneur en eau (Brie, 2018). Leur consommation prend une plus grande importance, à la fin de l'ancien régime, grâce à l'arrivée en quantité du sucre de canne en provenance des Antilles, favorisée par l'essor de l'esclavage (Walvin, 2020). Au XIX ${ }^{\text {eme }}$ siècle en Europe, la transition du sucre de canne à celui de betterave, produit localement, finit d'en banaliser la consommation (France Pittoresque, 2016) par la disparition du coût d'acheminement et l'augmentation des quantités produites.

\section{Conservation par l'acidification ou baisse de la valeur du potentiel hydrogène}

Généralités sur le pH ou potentiel hydrogène. Le $\mathrm{pH}$ de la majeure partie des aliments est compris entre 4,5 et 7 , avec des valeurs extrêmes allant de $\mathrm{pH} 2$ pour le vinaigre à $\mathrm{pH} 8$ pour le blanc d'œuf cru. Pour étudier ce paramètre, il faut prendre en compte son effet sélectif sur chaque germe, qui n'aura d'activité métabolique que dans une fourchette de valeurs de $\mathrm{pH}$ (minimum, maximum) qui lui est propre :

- Germes acidophiles (flore lactique, levures, moisissures)

- Germes neutrophiles (très nombreux)

- Germes basophiles (Bacillus, clostridies ...) de putréfaction, inhibés par les $\mathrm{pH}$ acides

\begin{tabular}{|lcc|}
\hline Flore pathogène & $\mathrm{pH}$ minimum & $\mathrm{pH}$ maximum \\
\hline Escherichia coli & 4,2 & 9,3 \\
\hline Salmonella typhi & 4 & 9,8 \\
\hline Clostridium botulinum & 4,8 & 8,5 \\
\hline Listeria monocytogenes & 4 & 9,6 \\
\hline Staphylococcus aureus & 4,5 & 7,5 \\
\hline Vibrio cholerae & 5,4 & 10,2 \\
\hline Brucella melitensis & 6,3 & 8,5 \\
\hline Mycobacterium tuberculosis & 5,1 & 8,8
\end{tabular}




\begin{tabular}{|lcc|}
\hline Flore technologique & $\mathrm{pH}$ minimum & $\mathrm{pH}$ maximum \\
\hline Streptococcus lactis & 4,5 & 9,1 \\
\hline Lactobacillus acidophilus & 3,5 & 7,5 \\
\hline Acetobacter sp & 1,9 & 7,2 \\
\hline Levures & 2,1 & 8,2 \\
\hline Moisissures & 1,7 & 11,2
\end{tabular}

Tableau II : Valeurs de pH, minimum et maximum, de quelques germes d'intérêt sanitaire ou technologique, montrant l'effet inhibiteur sur la flore pathogène des valeurs de pH inférieures à 4 (Cuq, 2007).

Il faut, de même, prendre en compte l'effet modificateur sur le $\mathrm{pH}$ des aliments, réalisé par les germes des flores technologiques. En particulier, l'abaissement au-dessous de 4 de la valeur du $\mathrm{pH}$ par la flore lactique, garantit l'inhibition des flores protéolytiques alcalinisantes d'altération (notamment celle des clostridies) et d'une grande partie des germes responsables de toxi-infections alimentaires (Tableau II).

Modalités d'application. L'acidification peut être réalisée directement par l'incorporation de vinaigre (cornichons, cerises, champignons, légumes ...). Elle peut aussi être assurée par l'intervention de la flore lactique et être le facteur principal de conservation comme dans le yaourt, le fromage, le kéfir ou même la choucroute. L'acidification par la flore lactique, peut aussi jouer un rôle secondaire de conservation comme dans le cas des salaisons sèches.

La choucroute. A partir du XVII ${ }^{\text {ème }}$ siècle, cette conserve de chou est appelée " chou aigre " ou « chou compost " en français et "sürkrüt» ou « kompostkrüt » en alémanique alsacien (Montel, 2005). Si l'on se réfère aux situations énoncées dans l'introduction de cet article, la choucroute est un exemple où la ressource saisonnière est conservée après la récolte. C'est une préparation de chou cabus (Brassica oleracea var. capitata) émincé, salé (2,5\%), épicé, pressé en tonneau pour réaliser l'anaérobiose. Une bonde positionnée au bas du tonneau permet l'élimination régulière des jus produits au cours de la fabrication. Son pH s'abaisse à 3,5 (2,5\% d'acide lactique au final) sous l'effet de la flore lactique (Leuconostoc mesenteroïdes et Lactobacillus plantarum). La fermentation durait traditionnellement 8 semaines mais a été réduite de nos jours à 2 semaines par ajout de vinaigre. Outre son $\mathrm{pH}$ de 3,5, elle est riche en vitamine C. Au XVIII ${ }^{\text {ème }}$ siècle, elle est emportée par le capitaine Cook sur ses navires (Menessier, 2018) en raison de son effet préventif et curatif sur le scorbut.

Les produits laitiers. Ces produits, notamment le yaourt (Birlouez, 2017) et le fromage, constituent un exemple où la ressource en lait est encore intimement dépendante du cycle de reproduction des premières espèces laitières domestiquées (probablement des caprins vers 10000 ans av JC) et donc de celui des saisons (Rischkowsky \& Pilling, 2008).

\section{Conservation par une très forte teneur en lipides}

Généralités sur la méthode de conservation en milieu gras. Cette méthode traditionnelle s'applique aux produits conservés dans l'huile comme les légumes ou les champignons, mais aussi aux viandes confites dans la graisse de porc ou de palmipèdes gras. L'utilisation d'un substrat très riche en lipides, constitue une "impasse " métabolique pour les bactéries. Elle est due à l'abaissement du pH de leur cytoplasme, provoqué par le démasquage des groupements acides des acides gras, au cours de leur dégradation. Le rétablissement d'un $\mathrm{pH}$ cytoplasmique normal, nécessite l'activation des pompes ioniques membranaires des bactéries, consommatrices d'énergie. Il s'en suit un bilan énergétique négatif qui, très rapidement, inhibe l'activité métabolique des bactéries (Rozier et al. 1985). Compte tenu de son rôle historique dans la conquête du continent nord-américain par les européens, nous développerons dans les lignes qui suivent l'exemple du pemmican des amérindiens (Thomas \& Ronnefeldt, 1977).

Le pemmican : caractéristiques et propriétés. Le nom " pemmican " signifie " graisse préparée " pour les Algonquiens ou les indiens Cris. Si l'on se réfère aux situations énumérées en introduction, le pemmican permet en tant que ressource alimentaire d'accompagner des déplacements au loin. La conservation du pemmican, qui est très prolongée, se compte en mois et même en années. Il peut être consommé en l'état ou bouilli, sur de grandes périodes, avec un impact limité des carences, grâce aux baies qui entrent dans sa composition et qui sont riches en vitamines A et $\mathrm{C}$. Le pemmican, préparé avec les viandes et les graisses les plus médiocres, sert à l'alimentation des chiens, animaux de bât des amérindiens, avant l'arrivée du cheval introduit en Amérique par les Européens. Le pemmican (Germain, 2003) est constitué, pour sa partie protéique, de viande de bison (Bison bison), de wapiti (Cervus canadensis), d'orignal (Alces americanus), de caribou (Rangifer trandus) ou d'ours (Ursu arctos), séchée au soleil ou au feu. La graisse et la moelle des os de la carcasse, constituant la partie lipidique du produit, sont chauffées puis mélangées à la viande séchée qui a été préalablement réduite en poudre. Des herbes et des fruits, pourvoyeurs de vitamines et de glucides, sont ajoutés à chaud à la préparation : canneberges (Vaccinium oxycoccus), bleuets (Vaccinium myrtilloïdes), plaquebières (Rubus chamaemorus), cerises et baies d'amélanchier (Amelanchier canadensis). Le pemmican est enfin façonné en boulettes légères, énergétiques, nourrissantes et équilibrées. Parmi les baies utilisées, la plaquebière, ou " plat de bièvre " ou ronce des tourbières, est particulièrement intéressante par sa richesse en vitamine $\mathrm{C}$ qui lui donne des propriétés préventives et curatives contre le scorbut. Cette baie contient, de plus, une forte teneur en acide benzoïque, qui présente un effet conservateur naturel qu'elle confère au pemmican (Rochefort \& Lapointe, 2003).

Quelques données historiques sur le pemmican. Le pemmican est un produit spécifique de la culture amérindienne des tribus du nord du continent. Des produits comparables sont connus en Mongolie et on peut penser que le pemmican faisait partie de l'alimentation des premiers amérindiens qui ont traversé le détroit de Behring, alors émergé par la dernière glaciation dite de Würm, pour 
venir peupler le continent américain. Pour les amérindiens, le pemmican est un aliment de voyage, utilisé pour les expéditions de chasse ou de guerre, mais c'est aussi un aliment de survie pendant les périodes de disette. Il devient, Pour établir ce bilan de façon synthétique, nous nous référerons au même titre que les pelleteries, un objet majeur de troc à la méthode de gestion globale de l'hygiène des aliments, avec les européens. Les coureurs des bois, premiers explora- présentée en 2013 dans un précédent article publié dans le teurs français du continent nord-américain, vont l'adopter Bulletin de l'Académie Vétérinaire de France (Bonne, 2013a). très vite, comme le feront ensuite les missions militaires Cette méthode s'articule autour du mécanisme d'occurrence d'exploration du continent, telles que celle de Lewis et Clark des accidents alimentaires et propose, dans un système de de 1804 à 1806 (Le Bris, 1993). Il est ensuite utilisé un cadres de couleurs, l'ensemble des dispositions appliquées à temps par " l'US Army " et la "Royal Navy " et les expédi- l'heure actuelle, pour garantir la sécurité sanitaire des aliments. tions polaires. Jules Verne en fera dans ses romans, l'aliment A la fin du XVIII ème siècle, seule une petite partie de ces disposide ses aventuriers.

\section{Bilan des méthodes de conservation des aliments} à la fin du XVIII'ème siècle tions, avait été comprise et mise en œuvre (Figure 1).

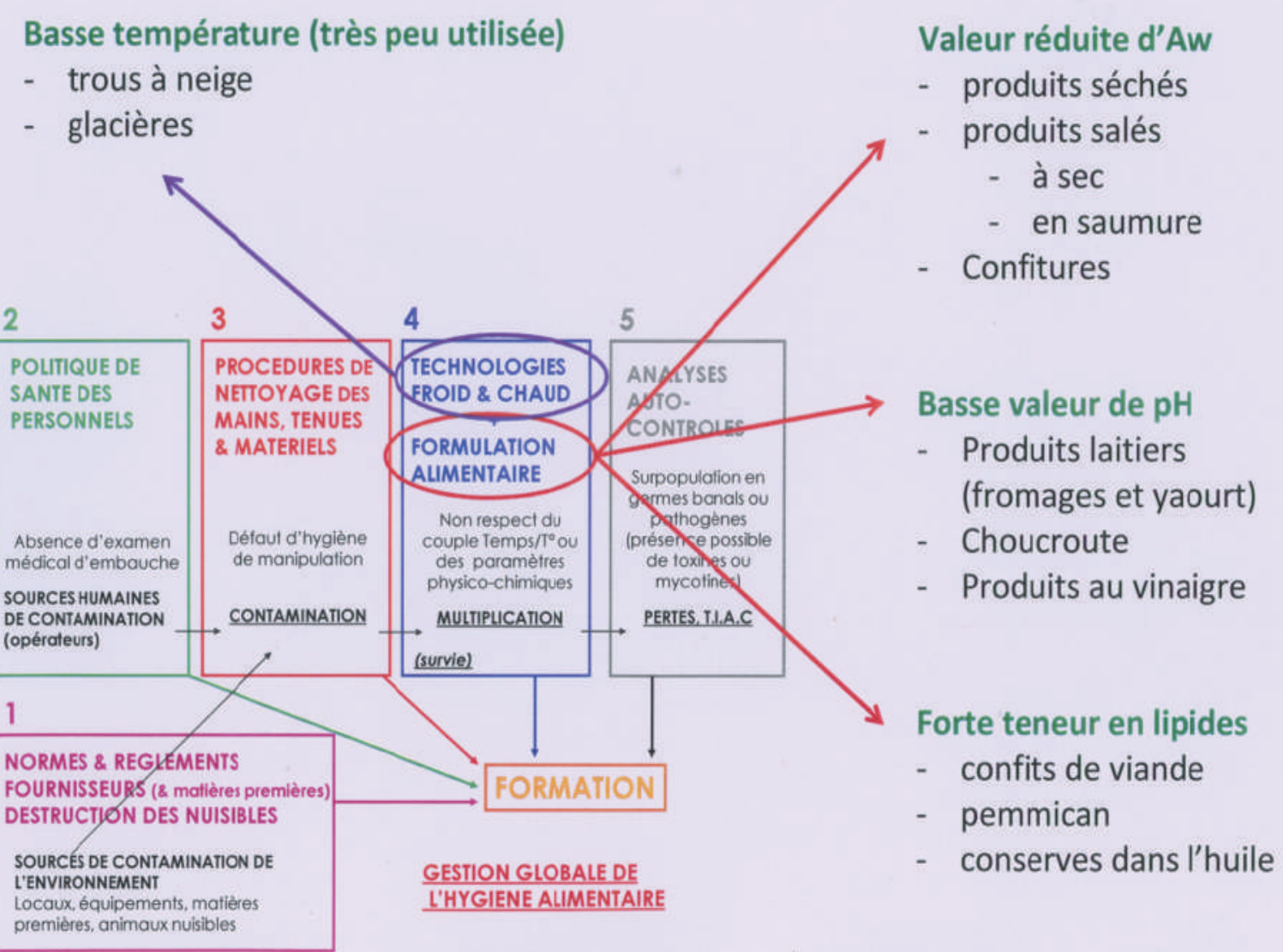

Figure $\mathrm{n}^{\circ} 1$ : Comparatif des méthodes de conservation des aliments, appliquées à la fin du XVIII ème siècle, avec l'approche actuelle de gestion globale de l'hygiène.

Cette figure montre de façon synthétique, qu'à part l'application très limitée de la chaîne du froid, les seules méthodes de conservation connues et utilisées à cette époque, reposaient sur une modification de la formulation des aliments, les rendant impropres à servir de substrats pour l'activité métabolique de multiplication des microorganismes.

\section{LE XIXème SIÈCLE ET L'ENTRÉE DANS L'ÈRE MODERNE DE LA CONSERVATION DES ALIMENTS}

Nous n'avons pas abordé dans cet article l'appertisation, la pasteurisation et les méthodes de conservation basées sur l'application du froid. Ces méthodes qui relèvent d'une approche scientifique, basée sur la gestion de paramètres physiques, le froid, la chaleur et le temps, ont fait entrer la conservation des aliments dans l'ère moderne, en abandonnant le modèle 
empirique au profit d'une approche expérimentale. Le suivi métronomique du froid des entrepôts frigorifiques ou celui de la température et de la durée de l'autoclavage d'appertisation, permet de garantir la salubrité des denrées qui y sont soumises. Les plans de maîtrise sanitaire (PMS) (Bonne et al. 2005) et la méthode HACCP " Hazard Analysis Critical Control Point " (Codex Alimentarius, 2003), ne sont que l'aboutissement actuel de ce changement de paradigme survenu au cours du XIX $^{\text {ème }}$ siècle.

\section{CONCLUSION}

Comme conséquence de ce nouveau paradigme, ne reposant pas sur la composition des aliments pour les rendre stables, un grand nombre de méthodes de conservation modernes, permettent de répondre à l'évolution des attentes des consommateurs qui souhaitent que leurs aliments présentent les caractéristiques de l'état frais. Contrairement aux méthodes traditionnelles, les méthodes modernes de conservation n'entrainent qu'une faible dénaturation des aliments, en particulier par l'application généralisée de la chaîne du froid, qu'il soit positif ou négatif. Le conditionnement des végétaux sous azote et le traitement du lait à ultra haute température (UHT), visent à conserver à ces produits les caractéristiques des denrées fraiches. D'autres méthodes aboutissent à ce résultat, parmi lesquelles peuvent être citées : l'irradiation, l'ozonisation ou les ultrahautes pressions. Elles permettent une décontamination partielle ou totale des aliments, sans provoquer l'effet dénaturant habituel des traitements par la chaleur. Toutes ces possibilités entraînent une très large diversification de l'offre alimentaire faite aux consommateurs, sous l'effet conjoint des méthodes de conservation et d'un marché devenu planétaire. Cependant, cette mondialisation des échanges engendre périodiquement de nouvelles crises sanitaires, en partie du fait de la conservation par le froid des agents pathogènes contenus dans les aliments, et malgré l'adoption au plan mondial des règles de sécurité sanitaire des aliments, établies par le Codex Alimentarius (FAO, OMS, OIE, CIPV) et imposées par l'OMC à ses pays membres (Bonne, 2013b).

$\mathrm{Au}$ plan géopolitique, certaines denrées alimentaires constituent toujours à l'heure actuelle des monnaies d'échange de haute valeur, dans un contexte mondial de très forte concurrence commerciale. Peut être évoquée à titre d'exemple la concurrence sur le marché de la crevette opposant les pays membres de l'ASEAN, les USA, l'Iran et Madagascar... Les différends entre l'UE et l'Amérique du nord (USA, Canada), portant sur les échanges de viande de bœuf contenant des résidus médicamenteux d'anabolisants et d'antibiotiques, en sont un autre exemple.

\section{BIBLIOGRAPHIE}

- Barré E. Petite histoire de la grande pêche. La Revue maritime. 2005 ; 474 : 1-7. Disponible à : http://ifm.free.fr/h tmlpages/pdf/2006/474histoiregrand epeche.pdf (consulté le 14/06/2021).

- Berche P. L'histoire du scorbut. Revue de biologie médicale. 2019 ; 347 : 49-59. Disponible à : https://www.revuebiol ogiemedicale.fr/images/Biologie_et_hi stoire/347-SCORBUT.pdf (consulté le 20/03/2021).

- Bernet E. Les grandes dates de la pêche à la morue 1497 - 1697, Extrait de la Bibliographie Francophone de la grande pêche. 1998. Disponible à : http://www.fecampterre-neuve.fr/Historique/Grande sDates.html (consulté le 05/03/2021).

- Birlouez E. Le yaourt un produit traditionnel en plein renouveau, CNIEL, Milk Factory ; 2017. Disponible à : http://ericbirlouez.fr/index.php/activites/articles/41-le-yaourt-un-produit-tr aditionnel-en-plein-renouveau (consulté le 11/02/2021).

- Bonne R, Camberou L, Wright N, Boccas F. Guidelines on GMP, GHP and HACCP for ASEAN Food SMEs, CEN/ASEAN editions, Djakarta, Indonesia. 2005, p 6. Disponible à : http://coli.usal.es/web/Guias/pdf/gu idelines HACCP UE.pdf (consulté le 11/02/2021).

- Bonne R. La gestion globale de l'hygiène dans les IAA : une méthode de facilitation pour la mise en ouvre des prescriptions d'hygiène du Codex Alimentarius. Bull Acad Vét France. 2013a ; 166 : 101-108.

- Bonne R. Présentation de deux méthodes originales visant à faciliter dans les IAA, la mise en ouvre de bonnes pratiques d'hygiène et de fabrication ainsi que de la méthode HACCP telle que définies par le Codex Alimentarius, Thèse de Doctorat d'Etat, Université Paul Sabatier, Toulouse, France. 2013b. Disponible à : http://thesesups. ups-tlse.fr/2161/ (consulté le 10/10/2021).

- Brie M. Histoire du pot de confiture (et de ce qu'on trouve dedans), in science et médecine, objets du quotidien. 2018. Disponible à : https://www.mariellebrie. com/histoire-du-pot-de-confitureet-de-ce-quon-trouve-dedans/ (consulté le 11/02/2021).

- Clouet A. L'histoire du biscuit de mer. 2010. Chroniques d'Histoire Maritime : 67. - Codex Alimentarius. Code d'usages international recommandé. Principes généraux d'hygiène alimentaire -
CAC/RCP 1, 2003. Disponible à : (http://www.codexalimentarius.n sset/download/standards/23/CXP _001f.pdf) (consulté le 11/02/2021).

- Cuq J-L. Cours de microbiologie alimentaire de l'Université Montpellier II, Département Sciences et Technologies des Industries Alimentaires ; 2007.

- Delâge D. Le pays renversé. Montréal : Éditions Boréal Express ; 1985 ; p 42.

- Rischkowsky B, Pilling D ; Origines et histoire de la diversité des animaux d'élevage, l'état des ressources zoogénétiques pour l'alimentation et l'agriculture dans le monde, édité par FAO. - Rome; 2008 ; part 1 ; p 7. Disponible à : http://www.fao.org/3/a1250f/a1250 f01.pdf (consulté le 29/06/2021).

- Fonssagrives J-B. Traité d'hygiène navale. Paris : Librairie J-B Baillères et Fils ; 1877 ; p 571.

- Frèrejean A. Découverte de l'appertisation par Nicolas Appert, Recueil de commémorations Nationales 2010, France Archives, Portail National des Archives de France ; 2010. Disponible à : https://francearchives.fr/fr/comm emo/recueil-2010/39492\#: :text= Soixante\%20ans\%20avant\%20Pa steur $\% 2 \mathrm{C} \% 20$ un, o\% $3 \%$ B $9 \% 2$ 
0son $\% 20$ p \%C3\%A8re\%20\%C3\%A 9tait\%20aubergiste (consulté le 10/02/2021).

- France Pittoresque. Coutumes et traditions, Confitures (Les) : une denrée de luxe devenue populaire en quelques siècles. France pittoresque édition ; 2016. Disponible à : https://www.france-pitt oresque.com/spip.php?article5815 (consulté le 10/02/2021).

- Germain HG. Les coureurs des bois. Paris : Éditions Libre Expression ; 2003 ; p 95.

- Hersart de la Villemarqué J. La pêche morutière française de 1500 À 1950, statistiques, climat et société. Plozané : Éditions Ifremer. 1995 ; p 21. Disponible à : https://archimer.ifremer.fr/doc/004 86/59783/62917.pdf (consulté le 11/02/2021).

- Le Bris M. Far West, journal de la première traversée du continent américain, la piste de l'ouest. Paris : Éditions Phébus ; 1993, p 159.

- Le Songeux. Le Scorbut, thèse de doctorat en médecine. Paris, 1827 ; p 10.

- Leys S. Les naufragés du Batavia. Paris : Éditions Points ; 2005 ; p 17.

- Mennessier M. Histoire de plantes : la choucroute et le scorbut, Le Figaro jardin, 2018. Disponible à : https://www.lefigaro.fr/jardin/ 2018/02/01/30008-20180201AR TFIG00287-histoire-de-plantes-lachoucroute-et-le-scorbut.php (consulté 11/02/2021).

- Montel MC, Béranger C, Bonnemaire J. Les fermentations au service des produits de terroir. Paris : INRA ; 2005, pp. 65-71.

- Olsen O. Quand la mafia pressait le citron sicilien. Courrier international, 19/10/2018, Paris.

- Oteng-Gyang K. Introduction à la microbiologie alimentaire dans les pays chauds. Paris : Éditions Lavoisier ; 1984, p 56.

- Portail pédagogique de l'Académie de Toulouse : Lind et le scorbut. 2021. Disponible à : https://disciplines.ac-to ulouse.fr/svt/sites/svt.disciplines.ac-to ulouse.fr/files/fichiers/ap/lind_et_le _scorbut.pdf (consulté le 29/06/2021).

- Rochefort L, Lapointe L. Production de petits fruits en tourbières, Groupe de recherche en écologie des tourbières, CRSNG, Université Laval, Québec ; 2007 ; p 35. Disponible à https://www .gret-perg.ulaval.ca/uploads/tx_centrerecherche/GUIDE_Petits_fruits_fr_ 2009_02.pdf. Consulté le 24/10/2021.

- Rozier J, Carlier V, Bolnot F. Bases microbiologique de l'hygiène des aliments. Maisons-Alfort : Éditeur École Nationale Vétérinaire, 1985.

- Soyez JM. Quand l'Amérique s'appelait Nouvelle-France. Paris : Éditions Fayard ; 1981, pp 7-8, 19, 35.

- Sweg S. Magellan. Paris : Éditions Grasset ; 1938 ; pp 217, 265, 245.

- Thomas D, Ronnefeldt K. Le peuple du premier homme. Paris : Éditions Flammarion; 1977 ; p 241.

- Walvin J. Histoire du sucre, histoire du monde. Paris : Éditions de la Découverte ; 2020. 